تاثير نوع المحراث في كثافة الادغال وخزين بذورها في التربة للمناطق الايمية بشمال العراق

\author{
خالد عصام العلاف \\ قسم المكائن و الالات الزر اعية/ كلية الزر اعة اعلة الغات

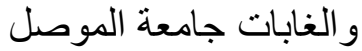

E-mail: Ahsultan2007@yahoo.com

احمد محمد سلطان

قسم المحاصيل الحقلية/كلية الزر اعة إنة

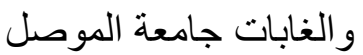

\title{
الخلاصة في
}

اجريت هذه الدراسة لتقييم نظم حراثة مختلفة في الحقول المبورة للمناطق متوسطة الامطار في

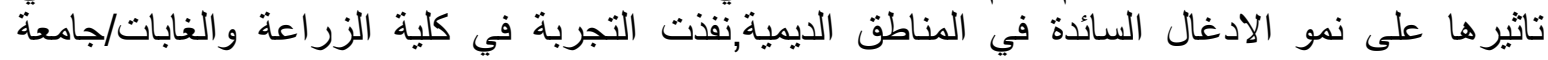

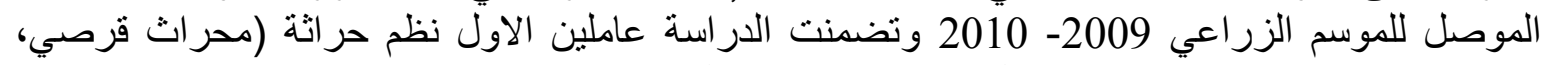

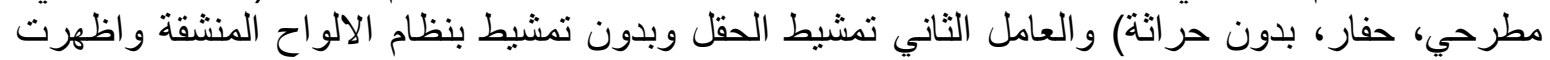

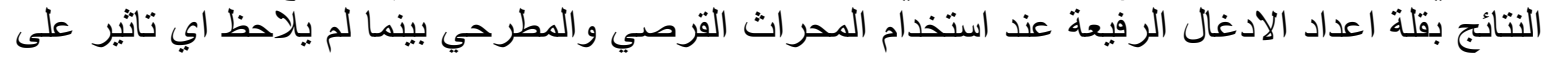

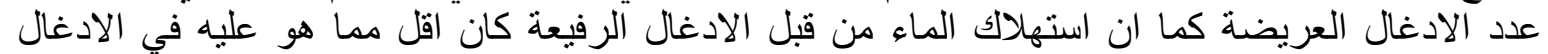

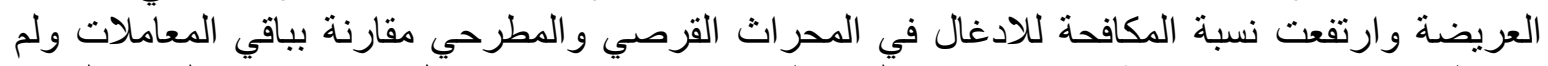

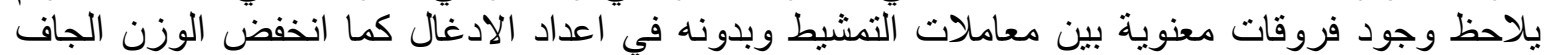

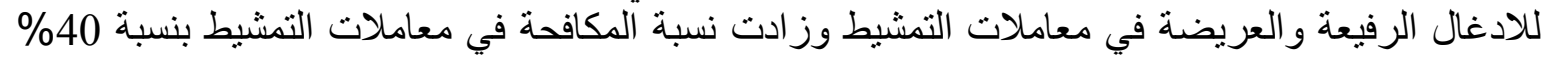

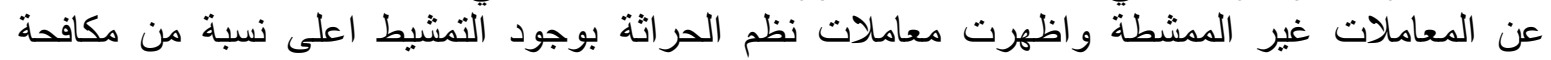

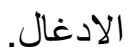

كلمات دالة: حراثات، خزين بذور، حقول مبورة. تاريخ تسليم البحث: 2011/9/8 وقبوله: 2012/6/18

\section{المقدمة}

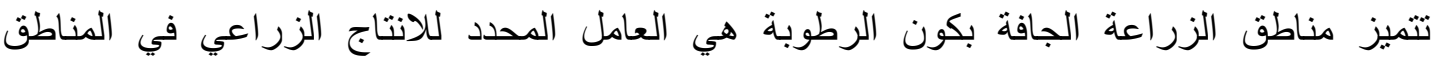

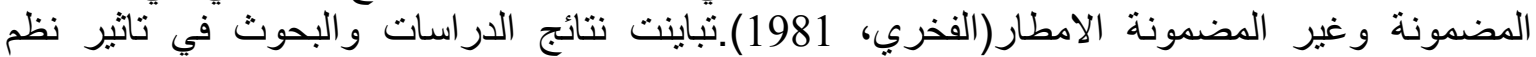

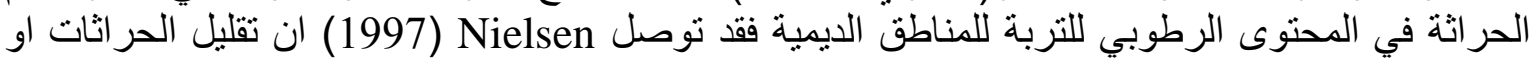

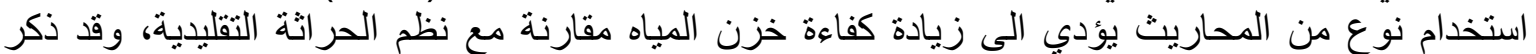
Bhashan

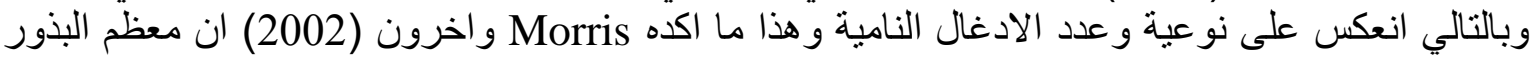

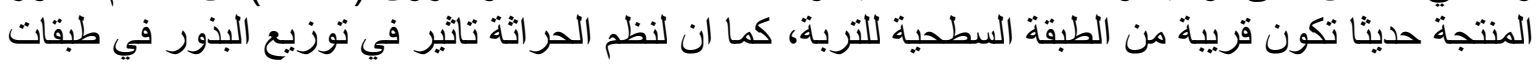

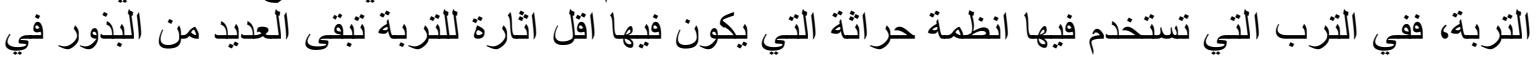

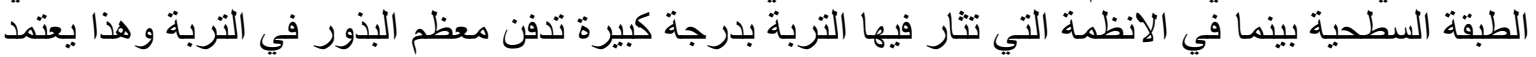

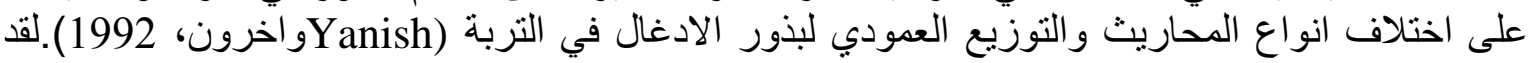

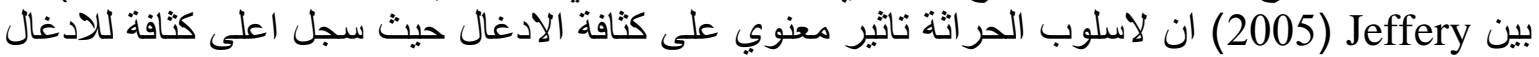

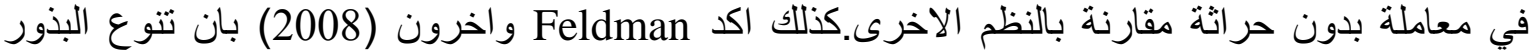

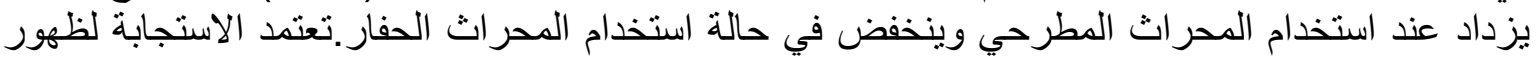

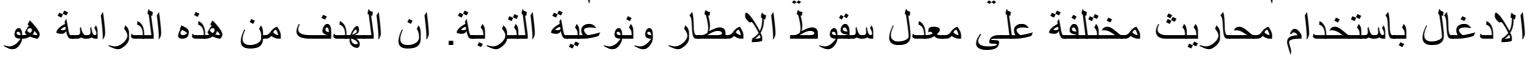
لمعرفة تاثير اختلاف نظم الحر اثة (المحروثة وغير المحروثة) للحقول المبورة على كثافة الادئة الادغال النامية في

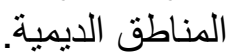

\section{مواد البحث وطرائقه}

اجريت تجربة حقلية في حقول كلية الزراعة والغابات/جامعة الموصل في الموسم الزراعي 2009-

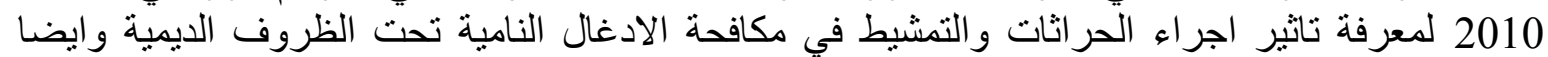

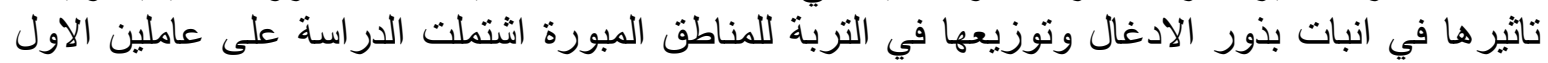

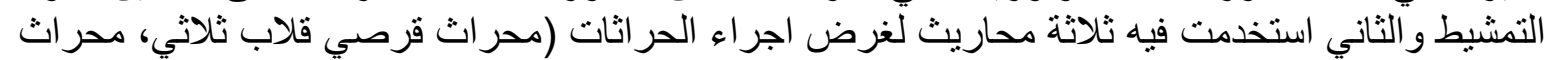

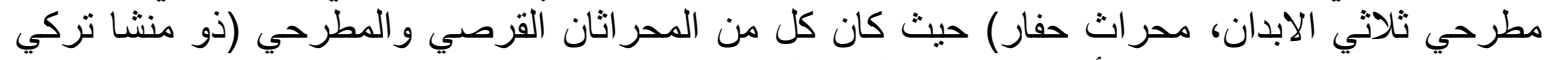

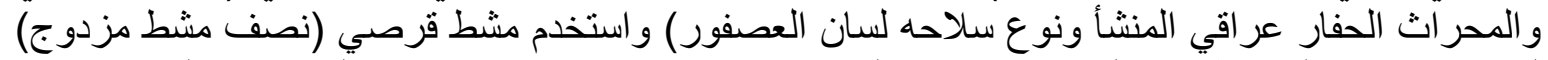
لغرض اجراء التمشيط بعد الحراثة اجريت الحراثة بتاريخ 2010/1/10 بجميع ألمحاريث الدذكورة وتم 
اجر اء التمشيط في اليوم التالي وتُركت معاملة بدون حر اثة لغرض المقارنة في حين تم تمشيط نصف معاملة

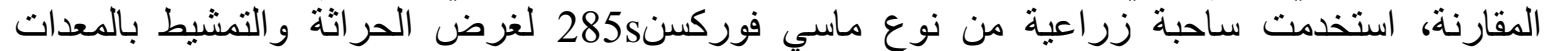

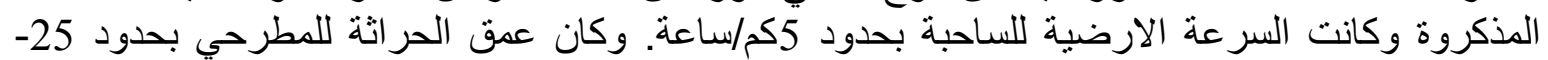

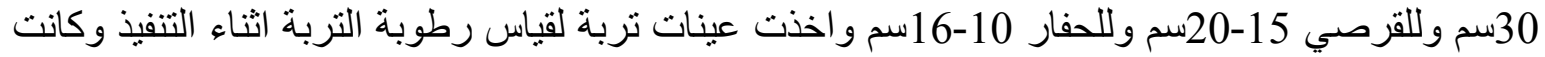

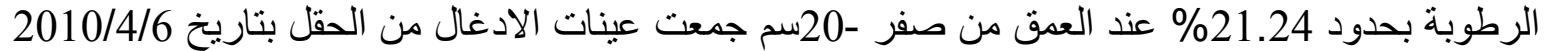

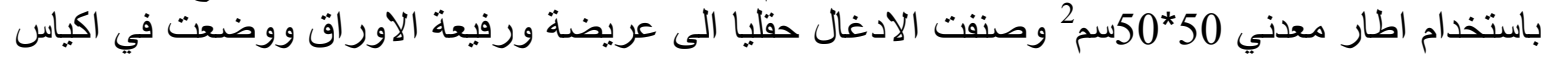

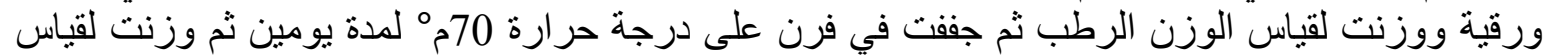

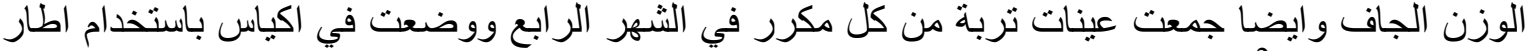

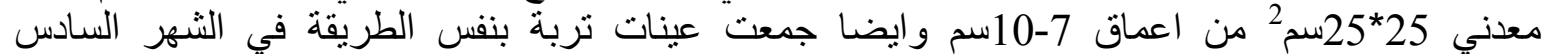

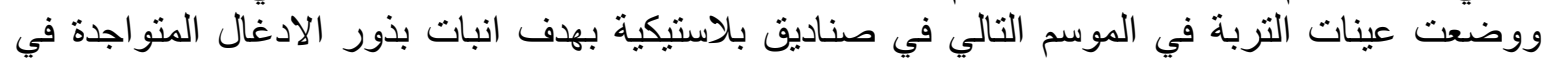

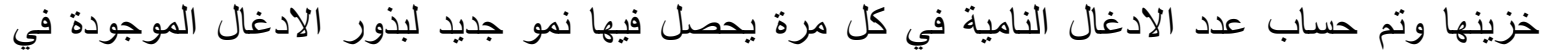

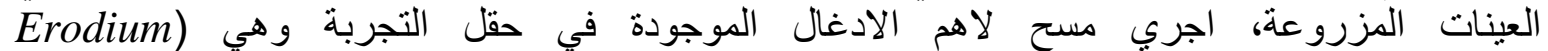
Raphanus منقار اللقلق، الثوفان البري . Avena fatua Lacoides(L) Willd.

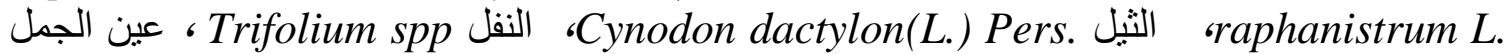

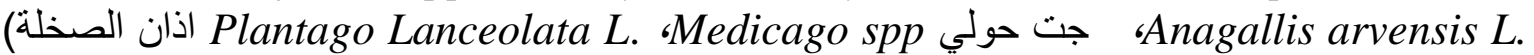

وتم حساب نسبة المكافحة باستخدام المعادلة التالية:-

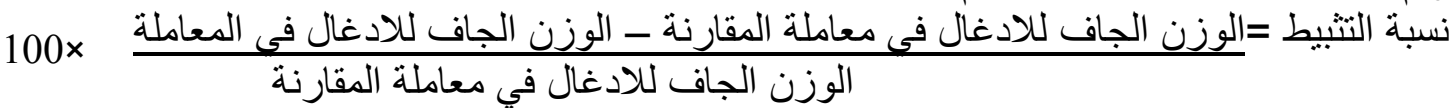

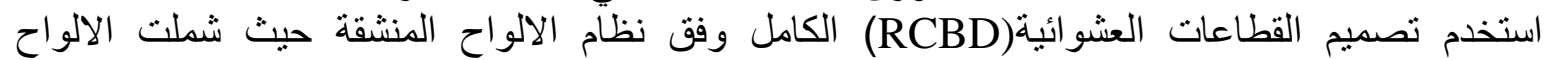

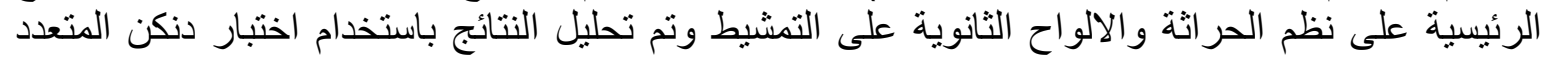
المدى و ادناه جدول السو اقط المطرية لثناء الموسم الزراعي.

\begin{tabular}{|c|c|c|c|c|c|c|c|c|}
\hline Oct. & Nov. & Dec. & Jan. & Feb. & Mar. & Apr. & May & $\begin{array}{c}\text { Average } \\
\mathrm{mm}\end{array}$ \\
\hline 24 & 35 & 75 & 32.5 & 60.5 & 62 & 15 & 17.5 & 312.5 \\
\hline
\end{tabular}

\section{النتائج والمتاقشة}

1 - تاثير انواع المحاريث في مكافحة الادغال:- ان من اهم العوامل المؤثرة في انبات بذور الأنئة الادغال

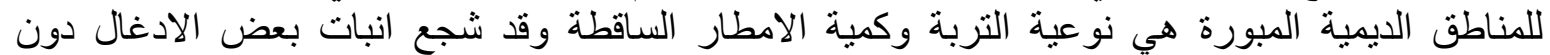

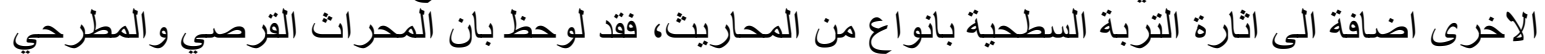

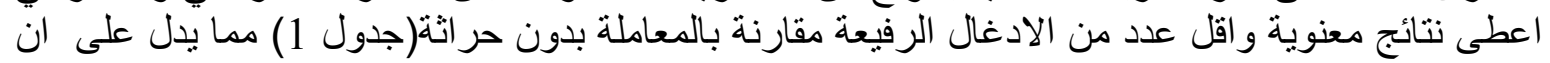

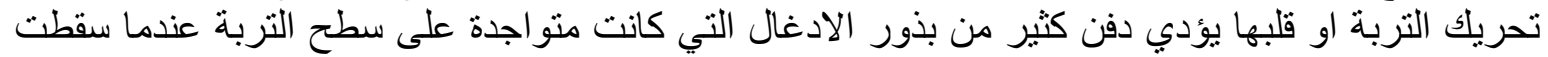

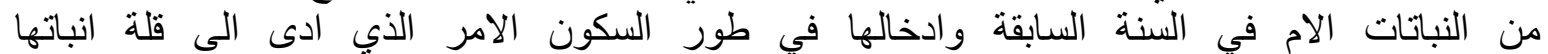

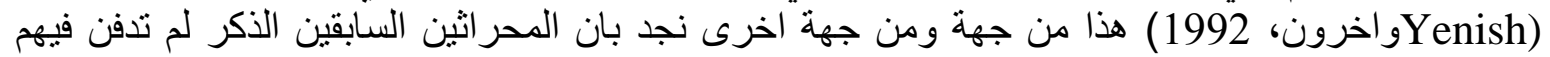

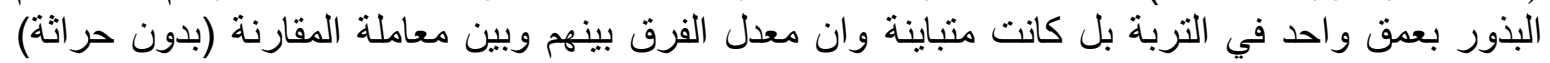

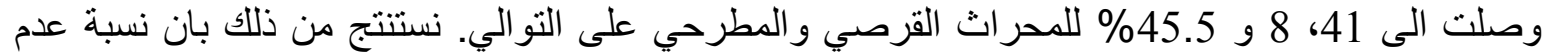
انبات بذور الادغال و المدفونة بالتربة وصلت الى 84.5 و 58.2\% للمحر اثين على التوالي وللتاكد من

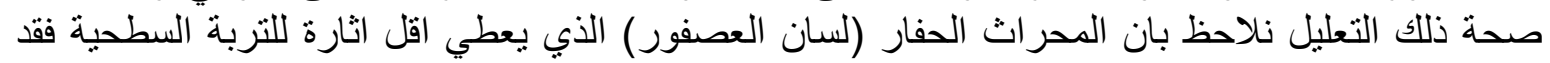

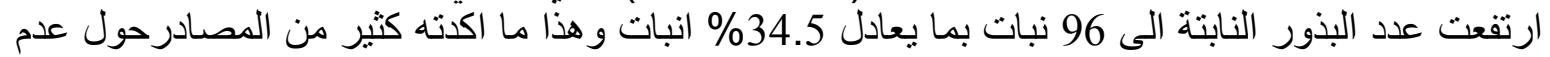

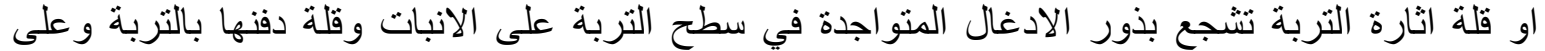

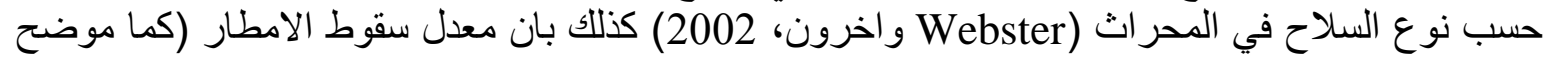

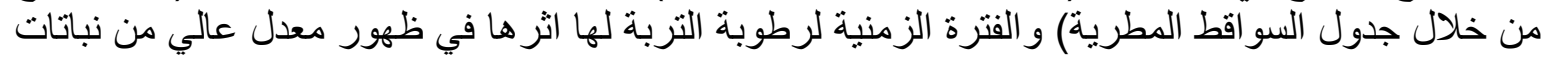

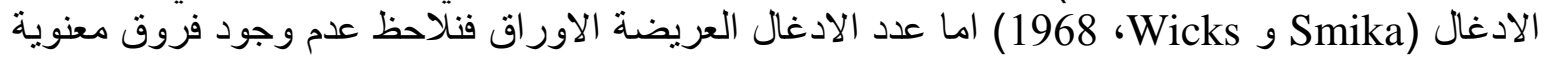

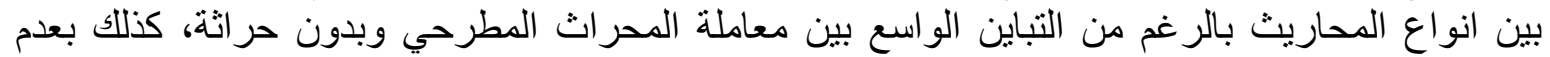

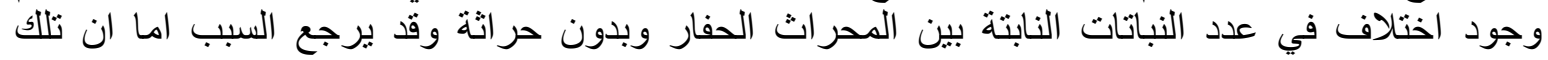

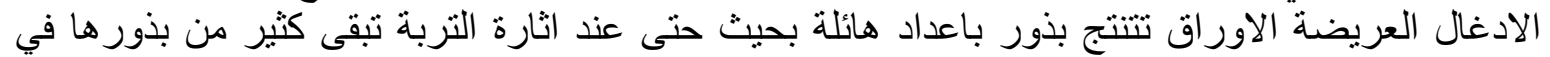

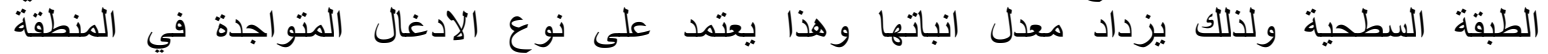

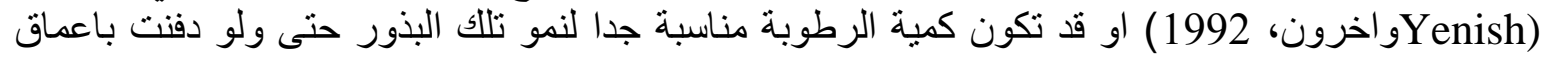


متباينة لقرة خزين المو اد الغذائية في البذور على انباتها وظهور ها فوق سطح التربة علما بان المحاريث

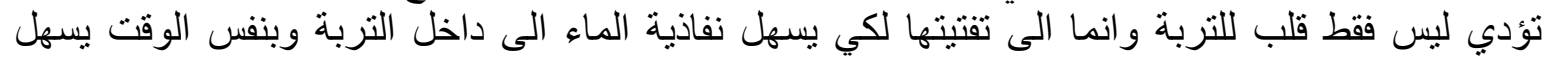

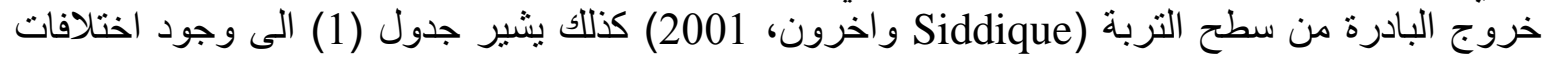
معنوية في الوزن الجاف والرطب لكلا النوعين من الادغال حيث اظهرت معاد معاملة المحراث القراث القردي

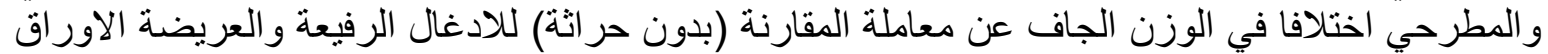

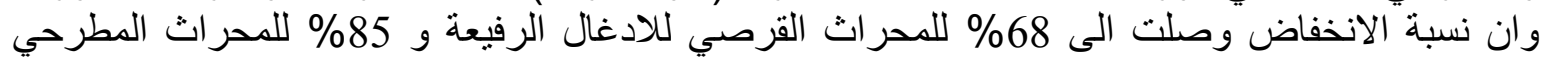

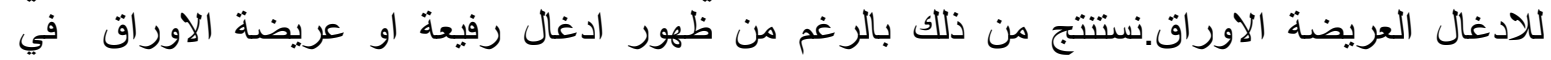

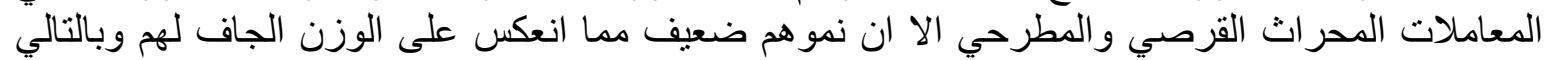

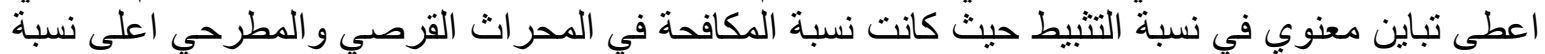

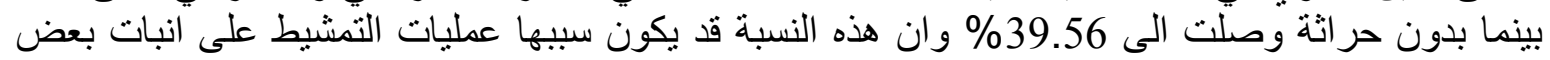

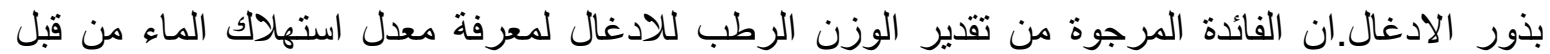

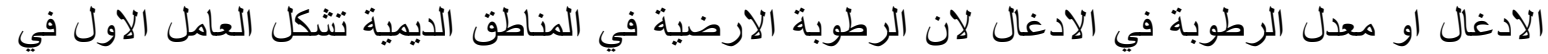

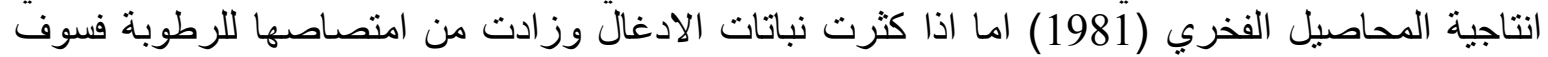

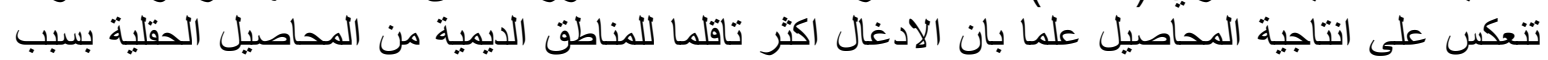

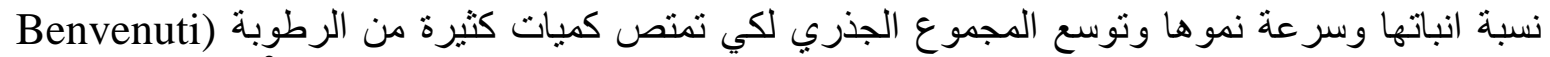

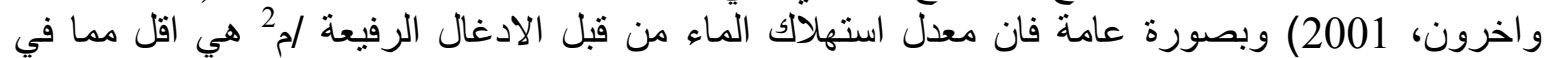

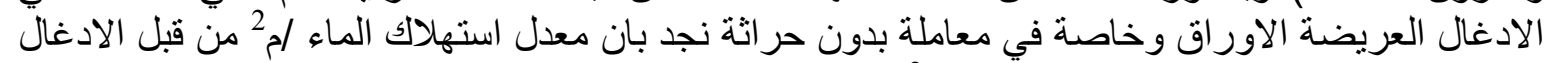

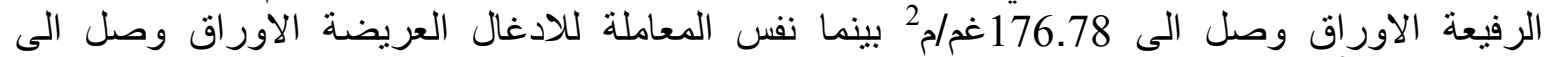

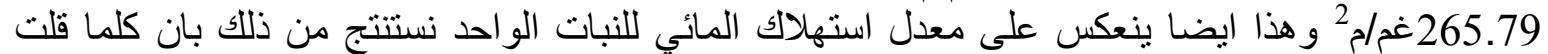

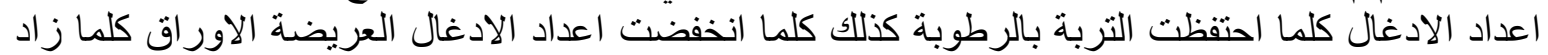

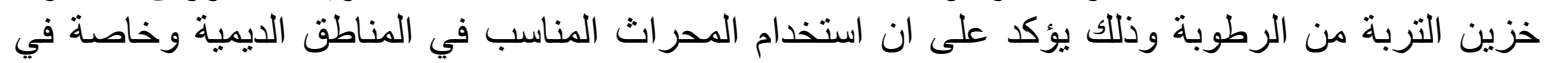
فترة التبوير يعطي نتائج جيدة ليس فقط في خفض الرئ اعداد الادغال و انما زيادة مخزون التربة من الرطوبة وخية الارضية.

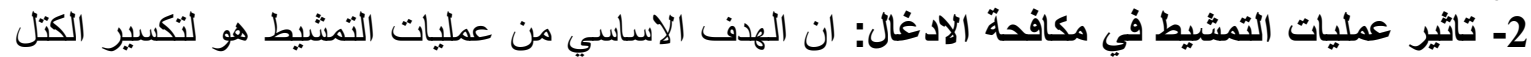

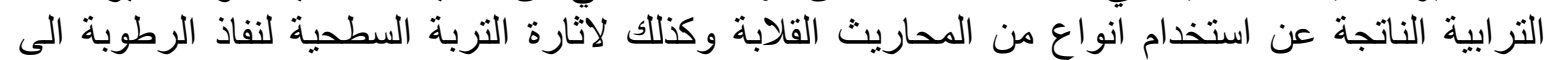

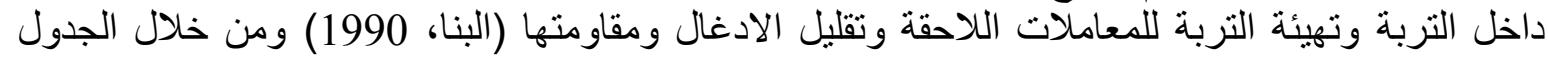

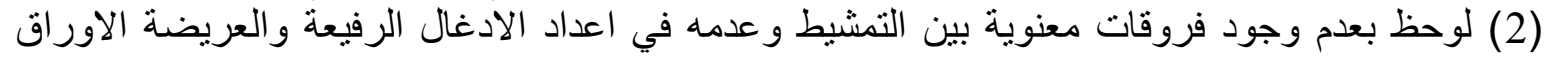

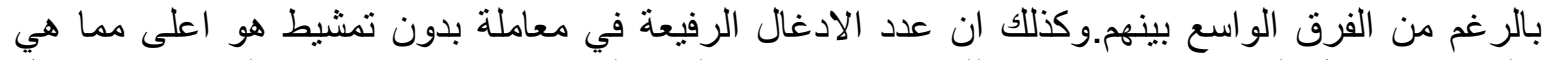

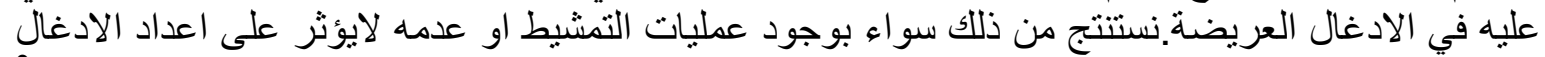

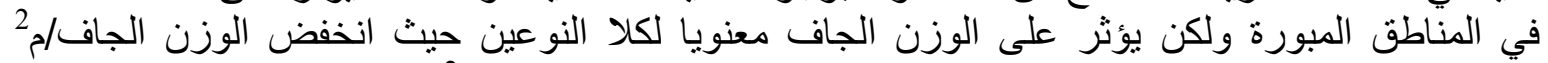

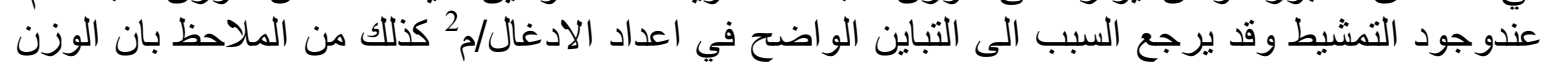

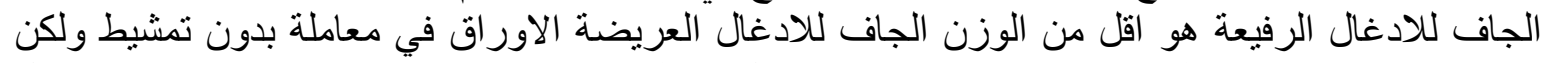

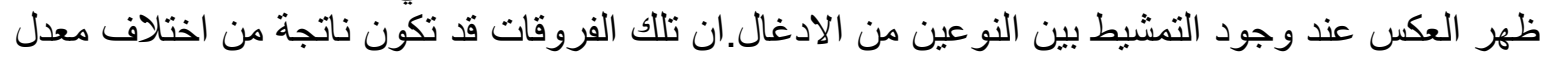

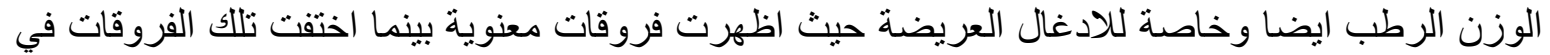

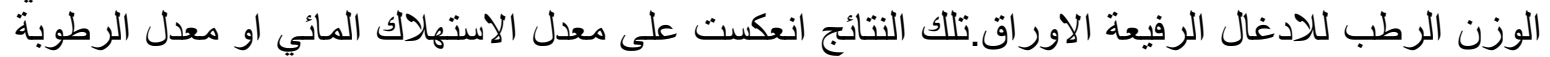

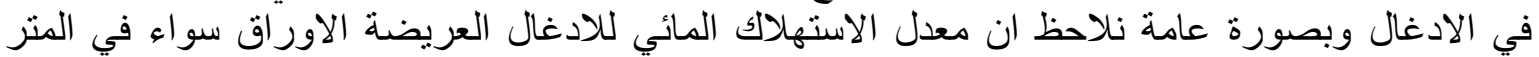

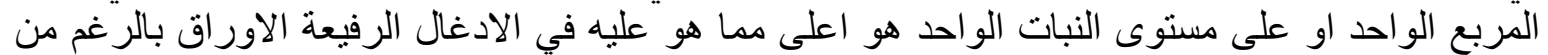

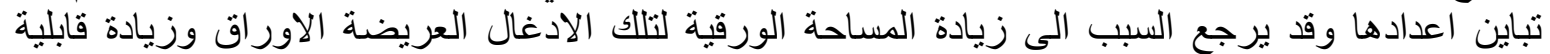

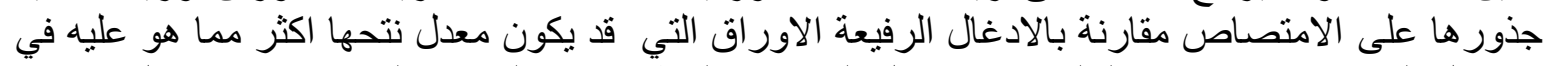

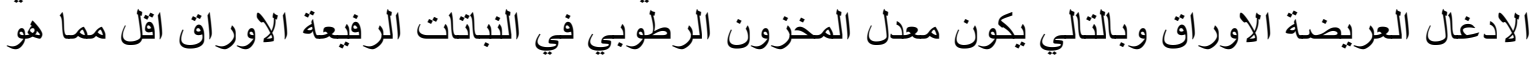

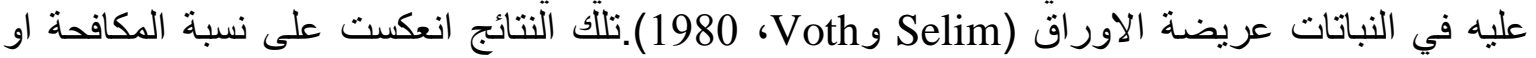
نسبة التثبيط وبصورة عامة نلاحظ ان عمليات التمشيط التي ادت الى قلّة اعداد الادغال زادت الته من نسبة

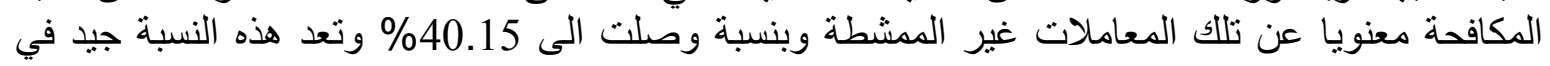
المناطق المناطق المطرية و هذه النتائج اكدها كثيرين من الباحثين منهم (Roberts و Feast، 1973).

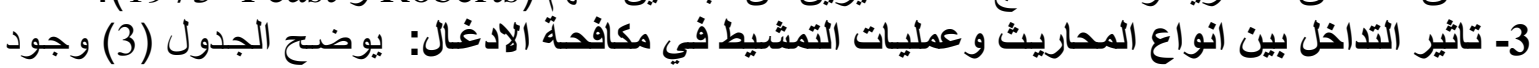

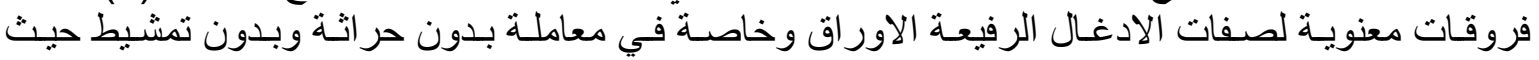

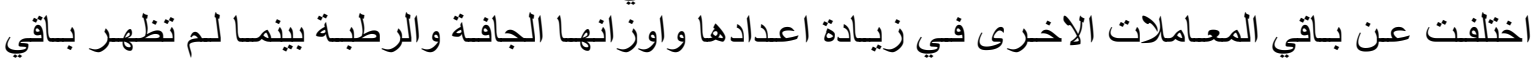

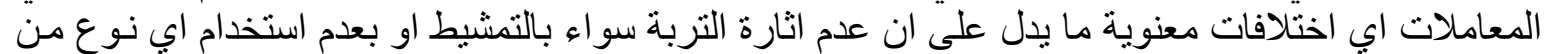


Mesopotamia J. of Agric.

Vol.(41) No.(4) 2013
ISSN:2224-9796(Online)

ISSN: 1815-316x(Print)

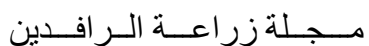

المجلد (41) العدد (4) 2013

المحاريث يزيد من نسبة انبات بذور الادغال الرفيعة وان استعمال المحاريث قد يقلل من اعدادها بسبب امـا

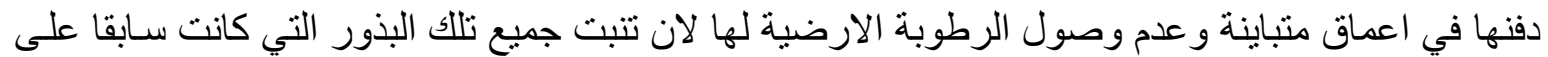

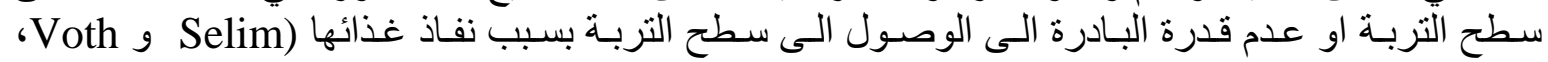

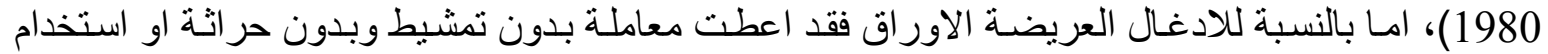

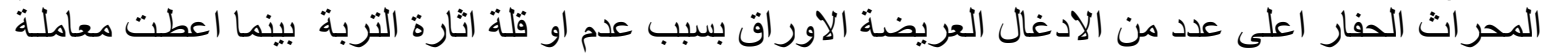

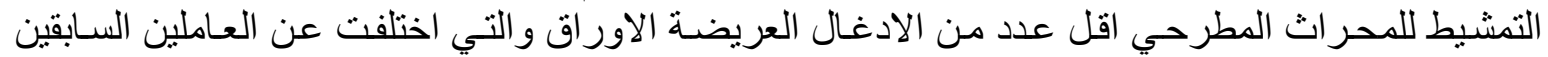

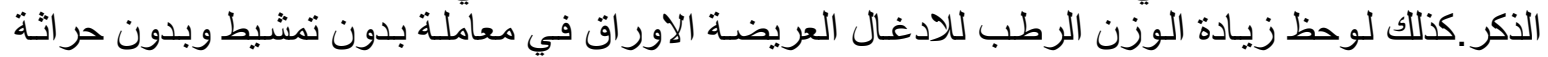

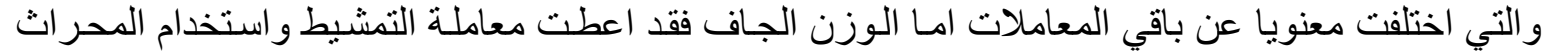

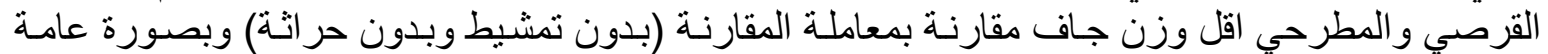

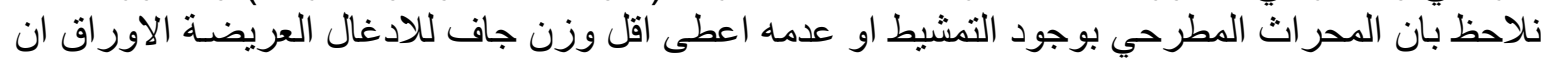

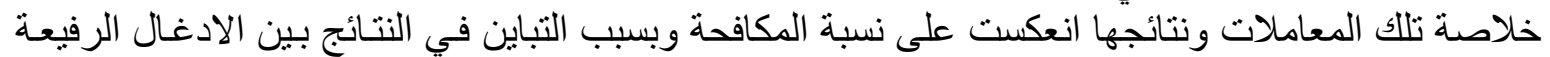

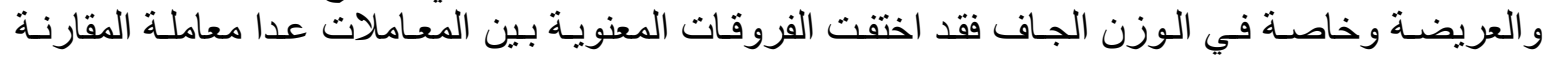

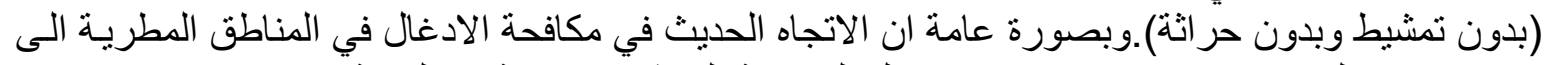

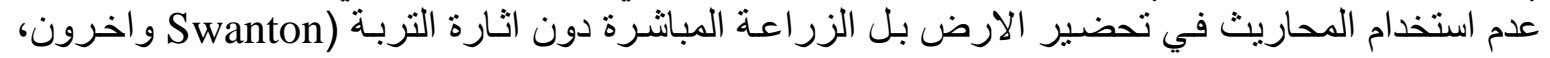

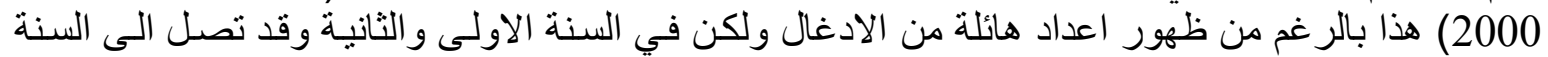

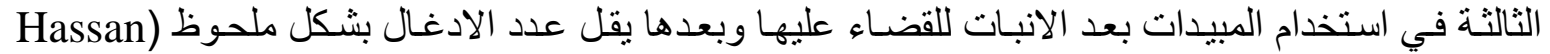

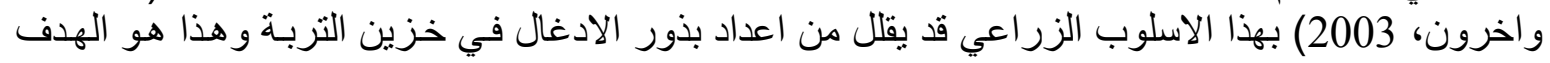

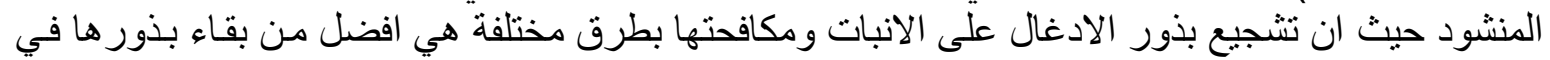

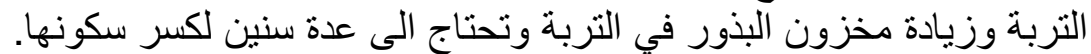

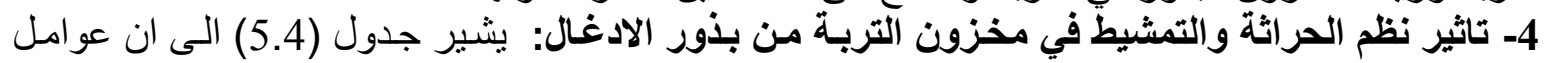

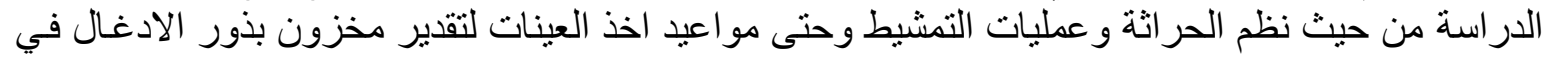

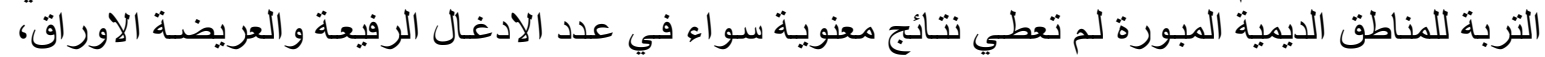

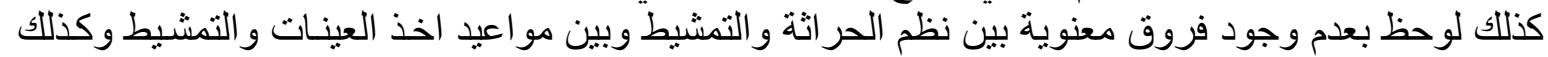

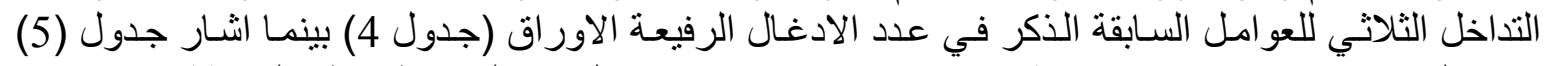

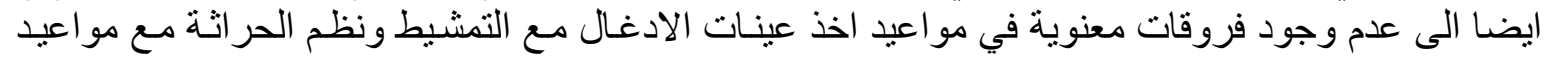

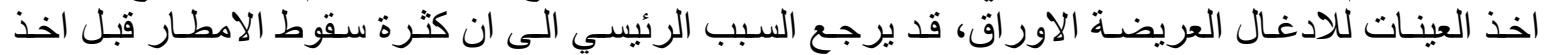

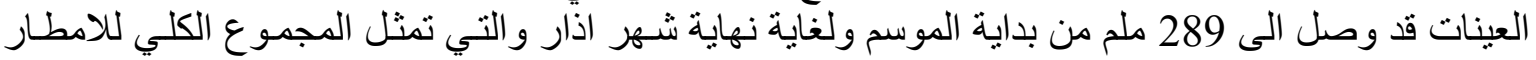

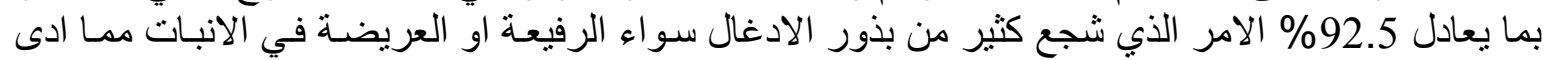

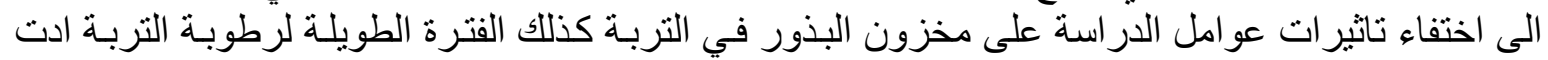

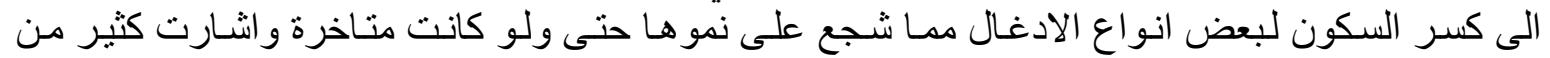

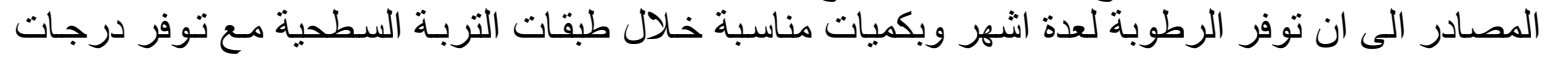

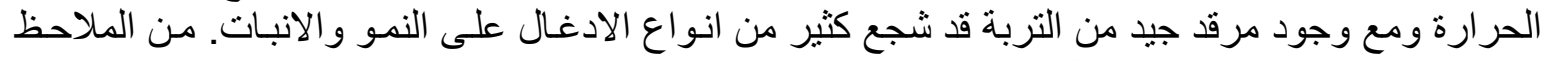

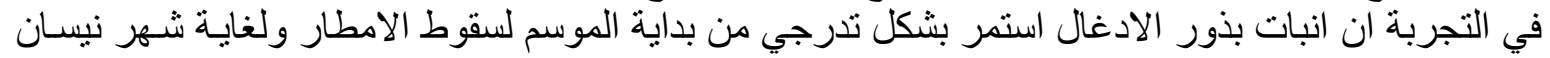

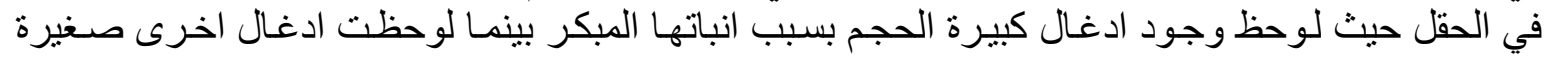

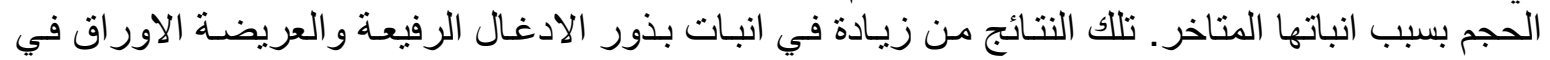

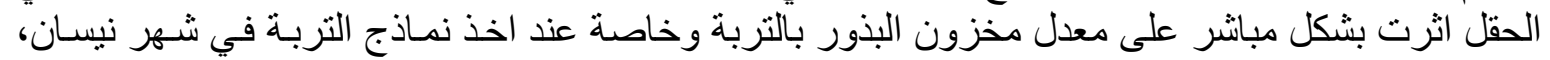

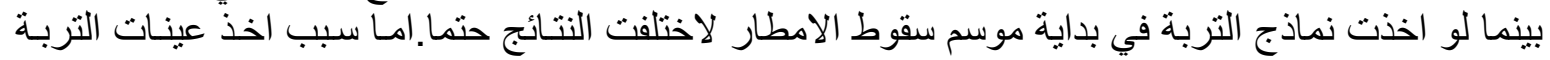

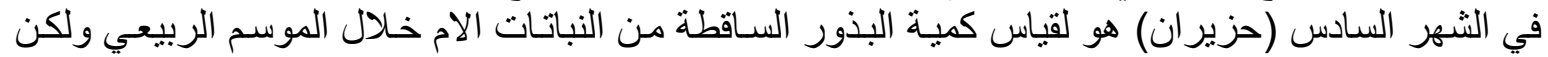

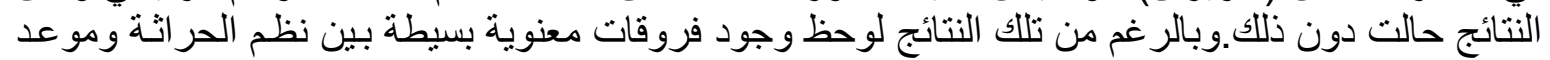

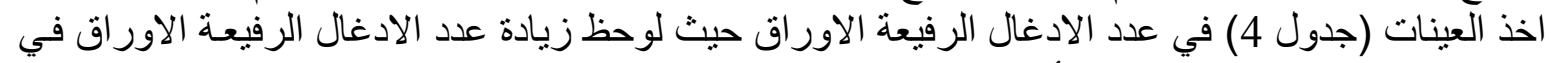

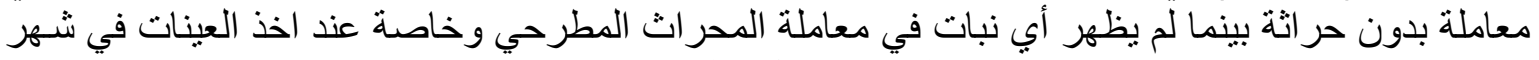

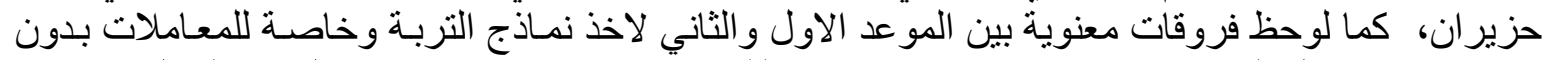

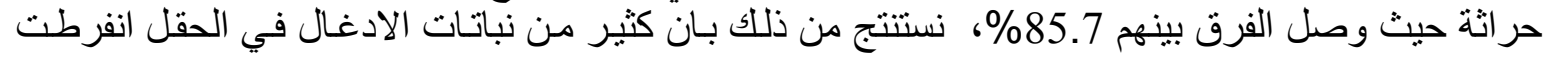

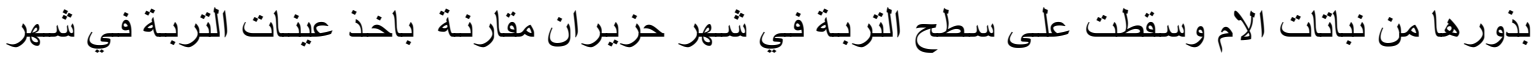

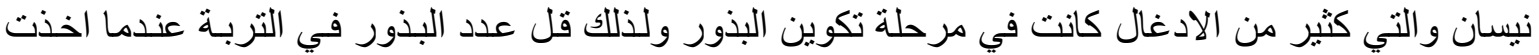

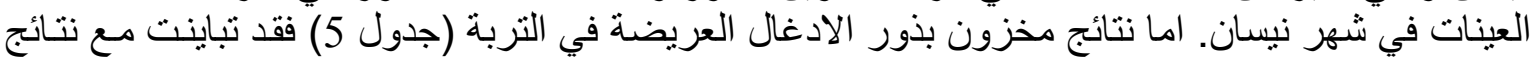

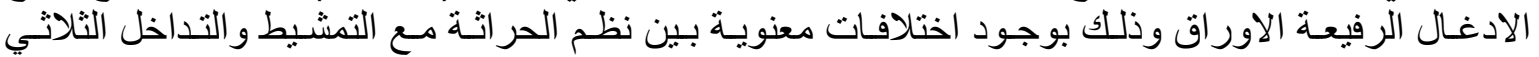
للعو امل الدر اسية وقد اظهرت الإن زيادة في عدد البذور المخزونة في التربة في معاملة بدون حر اثنة وبدون 
Mesopotamia J. of Agric.

Vol.(41) No.(4) 2013
ISSN:2224-9796(Online)

ISSN: 1815-316x(Print)
مـجــلة زر اعــة الـر افـدين

المجلد (41) العدد (4) 2013 
Mesopotamia J. of Agric.

Vol.(41) No.(4) 2013
ISSN:2224-9796(Online)

ISSN: 1815-316x(Print)
مـجــلة زر اعــة الـر افـدين

المجلد (41) العدد (4) 2013 
تمشيط وربما يرجع السبب الى بقاء البذور فوق سطح التربـة من العـام الماضي وكذللك من البذور المتكونـة

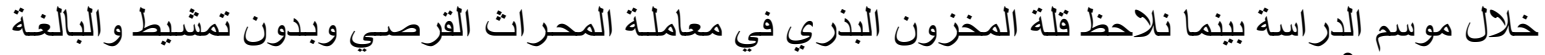

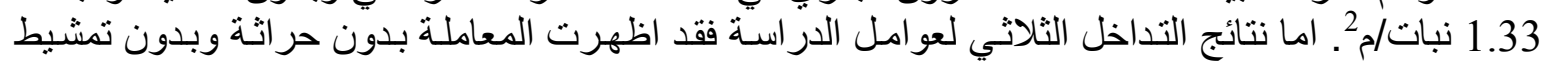

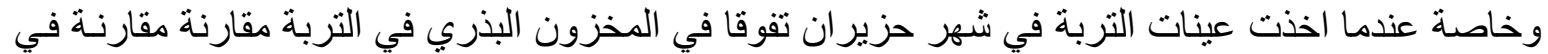

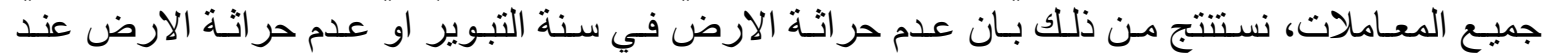

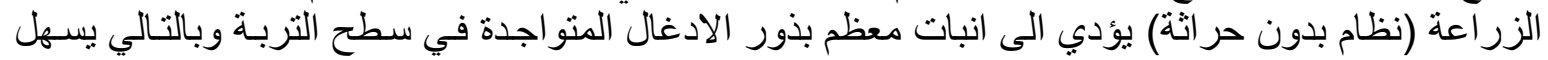

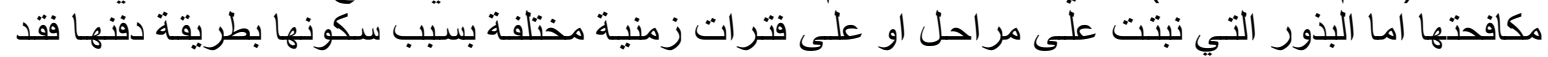
تشكل معضلة في عمليات المكافحة.

\section{TILLAGE TYPES EFFECT ON WEED DENSITY AND WEED SEED BANK UNDER DRY LAND FARMING IN NORTHERN IRAQ}

A.M. Sultan

Dep. Of Field Crop/College Of

Agriculture And Forestry, Univ. Of

Mosul
K.E. Al-allaaf

Dep.Of Agric. Machines \&

Equipments/College Of Agriculture And

Forestry,Univ.Of Mosul

E-mail: Ahsultan2007@yahoo.com

\section{ABSTRACT}

Weed control study was carried out in order to evaluate different plowing systems in dry land farming during fallow season and their effects on weed growth, The study was conducted in the College of Agriculture and Forestry/ Mosul university during 2009-2010 consisting of two factors, the first was plowing systems (disk plow, mold board plow, chisel, no-tillage) and the second factor harrowing and no harrowing after plowing. The results showed reducing the number of narrow leave weed in plowing by disk plow and mold board plow with no effects on number of broad leaved weed and water consumption by narrow leaved weed was less then broad leaved weed, The weed control percentage increased for disk plow and mold board plow comparison with other treatments .On the other hand no, significant effects were seen in number of weed with harrowing, also weed dry weight for both types of weed decreased and weed control percentage increased by $40 \%$ in harrow treatment in compression and no harrow. Plowing systems with harrowing showed highest weed control percentage.

Key Word: Tillage,Weed Seed Bank ,Farrow Field

Received: 11/9/2011 Accepted: $18 / 6 / 2012$

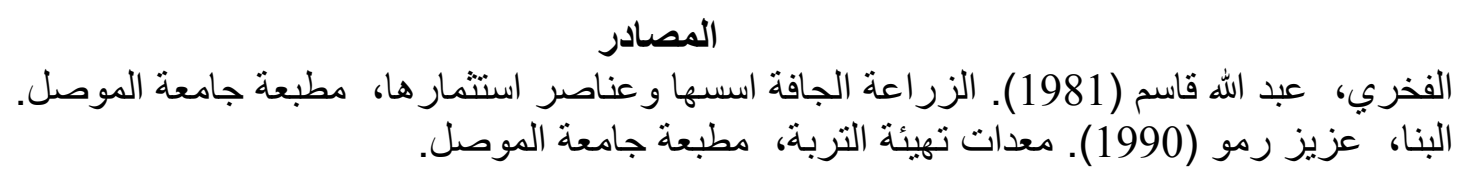

Bhushan, L. S.; S. B. Varade and G. P. Cupta (1973). Influence of tillage practices on cold size, porosity, and water retention. Indian Journal of Agriculture Science,43(5):466-471.

Benveuti,S.;M.Macchia and Mieje (2001).Quantitive analysis of emergence of seedling from buried weed seeds with increasing soil depth. Weed Science,49(4):528-535. 
Feldman,S.R.;C. Alzugaray ; P.S. Torres; P. Lewis (2008). The effect of different tillage systems on the composition of the seed bank. Weed Research, 37(2):71-76.

Hassan,G.; N. Khan and H. Khan (2003). Effect of zero tillage and herbicides on the weed density and yield of chickpea under rice-based condition of D.I.Khan.Weed Science Research, 9(3\&4):193-200.

Jeffery, S. C. (2005). Weed seed bank affected by tillage intensity for barley in Alaska. Soil Tillage Research, 90(1-2):156-161.

Morris,A.B.;R.S. Baucom and M.B.Cruzan (2002). Stratified analysis of the soil seed band in the cedar glade endemic(Astragalus bibullatus): evidence for historical change in genetic structure. American Journal of Botany, 89:2936.

Nielsen, D. C. (1997).Water use and yield of canola under dry land condition in the central great plains. Journal Production Agriculture, 10(2):307-313.

Roberts,H.A. and P.M. Feast(1973).Changes in the numbers of viable weed seeds in soil under different regimes. Weed Research,13:298-303.

Smika, D. E. and G. A. Wicks (1968). Soil water storage during fallow in a central great plains as influenced by tillage herbicide. Soil Science Of American Preceding, 32:591-594.

Siddique, K. H. M.; K. L. Regan; D. Tennant and B. D. Thomson (2001).Water use and water use efficiency of cool season grain legumes in low rainfull Mediterranean-type environments. European Journal of Agronomy, 15:267-280.

Selim, H. M. and R. D. Voth (1980). Soil water behavior under different tillage practices. Louisiana Agriculture, 23(3);14-15.

Swanton, C. J.; A. Shrestha; S. Z. Kenzevic; R.C.; Roy; B. R. Ball-Coelho (2000). Influence of tillage type on vertical weed seed bank distribution in a sandy soil. Canadian Journal of Plant Science, 80:455-457.

Webster, T. M.; J. Cardina and A. D. White (2002). Weed seed rain, soil seed banks and seedling recruitment in not tillage crop rotation. Weed Science, 51(4):569-575.

Yenish, J. P.; J. D. Doll and D. D. Buhler (1992). Effect of tillage on vertical distribution and viability of weed seed in soil. Weed Science, 40:429-433. 